\title{
On the stability of a functional equation deriving from additive and quadratic functions
}

Wang Liguang* and Li Jing

\section{"Correspondence:}

wangliguang0510@163.com School of Mathematical Sciences, Qufu Normal University, Qufu, Shandong 273165, China

\author{
Abstract \\ In this article, we investigate the Hyers-Ulam stability of the following functional \\ equation

$$
f(x+2 y)+f(x-2 y)=f(x+y)+f(x-y)+3 f(2 y)-6 f(y)
$$ \\ on quasi- $\beta$-normed spaces. \\ MSC: $45 \mathrm{~J} 05 ; 34 \mathrm{k} 30 ; 34 \mathrm{~K} 20$ \\ Keywords: additive mapping; quadratic mapping; quasi- $\beta$-normed spaces; \\ Hyers-Ulam stability
}

\section{Introduction}

The stability problem of functional equations originated from the following question of Ulam [1] concerning the stability of group homomorphisms:

Give a group $\left(G_{1}, *\right)$ and a metric group $\left(G_{2}, \cdot, d\right)$ with the metric $d(\cdot, \cdot)$. Given $\epsilon>0$, does there exists a $\delta>0$ such that iff: $G_{1} \rightarrow G_{2}$ satisfies $d(f(x * y), f(x) \cdot f(y))<\delta$ for all $x, y \in G_{1}$, then there is a homomorphism $g: G_{1} \rightarrow G_{2}$ with $d(f(x), g(x))<\varepsilon$ for all $x \in G_{1}$ ?

Hyers [2] gave the first affirmative partial answer to the question of Ulam for Banach spaces. Hyers's theorem was generalized by Aoki [3] for additive mappings and by Rassias [4] for linear mappings by considering an unbounded Cauchy difference. The study of Rassias has provided a lot of influence on the development of what we called the generalized Hyers-Ulam-Rassias stability of functional equations. In 1990, Rassias [5] asked whether such a theorem can also be proved for $p \geq 1$. In 1991, Gajda [6] gave an affirmative solution to this question when $p>1$, but it was proved by Gajda [6] and Rassias and Semrl [7] that one cannot prove an analogous theorem when $p=1$. In 1994, a generalization was obtained by Gavruta [8] who replaced the bound $\varepsilon\left(\|x\|^{p}+\|y\|^{p}\right)$ by a general control function $\phi(x, y)$. Beginning around 1980, the stability problems of several functional equations and approximate homomorphisms have extensively been investigated by many authors and there are many interesting results concerning this problem [9-22].

The functional equation

$$
f(x+y)+f(x-y)=2 f(x)+2 f(y)
$$


is called the quadratic function equation. Function $f(x)=a x^{2}$ satisfies (1.1). Every solution of (1.1) is called a quadratic mapping. Skof [9] solved the Hyers-Ulam stability problem of the quadratic functional equation in Banach spaces. Kim and Rassias [10] proved the stability of the Euler-Lagrange quadratic mappings. Park [11] considered the stability of quadratic mappings on Banach modules. Moslehian et al. [12] considered the approximation problem of quadratic functional equation on multi-normed spaces.

Hyers [2] considered the stability of the additive functional equation

$$
f(x+y)=f(x)+f(y) .
$$

Function $f(x)=d x$ satisfies (1.2). Every solution of (1.2) is called an additive mapping. The Cauchy type additive functional equation and its generalized Hyers-Ulam 'product-sum' stability have been studied in $[21,22]$.

In this article, we consider a new functional equation

$$
f(x+2 y)+f(x-2 y)=f(x+y)+f(x-y)+3 f(2 y)-6 f(y)
$$

deriving from the quadratic functional equation (1.1) and the additive functional equation (1.2). It is not difficult to check that $f(x)=a x^{2}+b x$ is a solution of (1.3).

The notion of quasi- $\beta$-normed space was introduced by Rassias and Kim [20]. This notion is similar to quasi-normed space. We fix a real number $\beta$ with $0<\beta \leq 1$ and let $\mathbb{K}=\mathbb{R}$ or $\mathbb{C}$. Let $X$ be a linear space over $\mathbb{K}$. A quasi- $\beta$-norm $\|\cdot\|$ is a real-valued function on $X$ satisfying the following conditions:

(1) $\|x\| \geq 0$ for all $x \in X$ and $\|x\|=0$ if and only if $x=0$.

(2) $\|\lambda x\|=|\lambda|^{\beta}\|x\|$ for all $\lambda \in \mathbb{K}$ and all $x \in X$.

(3) There is a constant $K \geq 1$ such that $\|x+y\| \leq K(\|x\|+\|y\|)$ for all $x, y \in X$.

The pair $(X,\|\cdot\|)$ is called a quasi- $\beta$-normed space if $\|\cdot\|$ is a quasi- $\beta$-norm on $X$. The smallest possible $K$ is called the modulus of concavity of $\|\cdot\|$. A quasi- $\beta$-Banach space is a complete quasi- $\beta$-normed space.

In the following, we recall some fundamental results in fixed point theory. Let $X$ be a set. A function $d: X \times X \rightarrow[0, \infty]$ is called a generalized metric on $X$ if $d$ satisfies

(1) $d(x, y)=0$ if and only if $x=y$;

(2) $d(x, y)=d(y, x)$ for all $x, y \in X$;

(3) $d(x, z) \leq d(x, y)+d(y, z)$ for all $x, y, z \in X$.

We also recall the following theorem of Diaz and Margolis [23].

Theorem 1.1 [23] Let $(X, d)$ be a complete generalized metric space and let $J: X \rightarrow X$ be a strictly contractive mapping with Lipschitz constant $0<L<1$. Then for each given element $x \in X$, either

$$
d\left(J^{n} x, J^{n+1} x\right)=\infty
$$

for all nonnegative integers $n$ or there exists a nonnegative integer $n_{0}$ such that

(1) $d\left(J^{n} x, J^{n+1} x\right)<\infty$ for all $n \geq n_{0}$;

(2) the sequence $\left\{J^{n} x\right\}$ converges to a fixed point $y^{*}$ of $J$;

(3) $y^{*}$ is the unique fixed point of $J$ in the set $Y=\left\{y \in X: d\left(J^{n_{0}} x, y\right)<\infty\right\}$; 
(4) $d\left(y, y^{*}\right) \leq \frac{1}{1-L} d(y, J y)$ for all $y \in Y$.

In 2003, Cadariu and Radu [24, 25] applied the fixed-point method to the investigation of the Jensen functional equation. By using fixed point methods, the stability problems of several functional equations have extensively been investigated by a number of authors (see [26-28]).

In this article, we will consider the solution and the Hyers-Ulam stability of the functional equation (1.3) on quasi- $\beta$-normed spaces using fixed point method.

\section{Solution of (1.3)}

We assume $X$ and $Y$ are real (or complex) linear spaces in this section.

Lemma 2.1 If an even function $f: X \rightarrow Y$ satisfies (1.3) for all $x, y \in X$, then $f$ is a quadratic mapping.

Proof Since $f$ is even, $f(-x)=f(x)$. Let $x=y=0$ in (1.3), we have $f(0)=0$. Let $x=0$ in (1.3), we have $f(2 y)=4 f(y)$ and therefore

$$
f(x+2 y)+f(x-2 y)=f(x+y)+f(x-y)+6 f(y)
$$

for all $x, y \in X$. Replace $x$ by $2 x$ in (2.1), we have

$$
f(2 x+2 y)+f(2 x-2 y)=f(2 x+y)+f(2 x-y)+6 f(y)
$$

for all $x, y \in X$. Replace $y$ and $x$ by $x$ and $y$ in (2.1), respectively, we have

$$
f(2 x+y)+f(2 x-y)=f(x+y)+f(x-y)+6 f(x)+6 f(y)
$$

for all $x, y \in X$ since $f$ is even and $f(2 y)=4 f(y)$. It follows from (2.2) and (2.3) that

$$
f(x+y)+f(x-y)=2 f(x)+2 f(y) .
$$

Hence $f$ is a quadratic mapping.

Lemma 2.2 If an odd function $f: X \rightarrow Y$ satisfies (1.3) for all $x, y \in X$, then $f$ is an additive mapping.

Proof Since $f$ is odd, we have $f(-x)=-f(x)$. Let $x=y=0$ in (1.3) we have $f(0)=0$. Let $x=0$ in (1.3), we have

$$
f(2 y)=2 f(y)
$$

for all $x, y \in X$. Thus for all $x, y \in X$, we have

$$
f(x+2 y)+f(x-2 y)=f(x+y)+f(x-y) .
$$


Replace $x$ by $2 x$ in (2.5), we have

$$
2 f(x+y)+2 f(x-y)=f(2 x+y)+f(2 x-y) .
$$

Replacing $y$ and $x$ by $x$ and $y$ in (2.5), respectively, we have

$$
f(2 x+y)-f(2 x-y)=f(x+y)-f(x-y) .
$$

It follows from (2.6) and (2.7) that

$$
2 f(2 x-y)=f(x+y)+3 f(x-y) .
$$

Replacing $y$ by $x-y$ in (2.8), we have

$$
2 f(x+y)=f(2 x-y)+3 f(y)
$$

for all $x, y \in X$. Hence,

$$
3 f(2 x-y)=3 f(x+y)+3 f(x-y)-3 f(y) .
$$

Replacing $y$ by $-y$ in (2.10), we have

$$
3 f(2 x+y)=3 f(x-y)+3 f(x+y)+3 f(y) .
$$

By (2.7), (2.10), and (2.11), we have

$$
3 f(x+y)-3 f(x-y)=6 f(y) .
$$

Replacing $y$ and $x$ by $x$ and $y$ in (2.12), respectively, we have

$$
3 f(x+y)+3 f(x-y)=6 f(x) .
$$

It follows from (2.12) and (2.13) that

$$
f(x+y)=f(x)+f(y)
$$

for all $x, y \in X$. Hence, $f: X \rightarrow Y$ is an additive mapping.

Theorem 2.1 A function $f: X \rightarrow Y$ satisfying (1.3) for all $x, y \in X$ if and only if there exist a symmetric bi-additive mapping $B: X \times X \rightarrow X$ and an additive mapping $A: X \rightarrow Y$ such that $f(x)=B(x, x)+A(x)$ for all $x \in X$.

Proof If there exist an symmetric bi-additive mapping $B: X \times X \rightarrow X$ and an additive mapping $A: X \rightarrow Y$ satisfying $f(x)=B(x, x)+A(x)$ for all $x \in X$, then it is not difficult to check that

$$
f(x+2 y)+f(x-2 y)=f(x+y)+f(x-y)+3 f(2 y)-6 f(y)
$$


for all $x, y \in X$. Conversely, let

$$
\begin{aligned}
& f_{e}(x)=\frac{f(x)+f(-x)}{2}, \\
& f_{o}(x)=\frac{f(x)+f(-x)}{2} .
\end{aligned}
$$

Then

$$
f(x)=f_{e}(x)+f_{o}(x)
$$

for all $x \in X$. It is not difficult to check that $f_{e}$ and $f_{0}$ satisfying (1.3). It follows from Lemmas 2.1 and 2.2 that $f_{e}$ and $f_{0}$ are quadratic and additive mappings, respectively. Hence, there exist a symmetric bi-additive mapping $B: X \times X \rightarrow X$ such that $f_{e}(x)=B(x, x)$ for all $x \in X$. Let $A(x)=f_{o}(x)$. Then we have $f(x)=B(x, x)+A(x)$ for all $x \in X$.

\section{The stability of functional equation (1.3)}

Now we consider the stability of the functional equation (1.3) using fixed point method.

In this section, we always assume that $X$ is a complex (or real) linear space and $Y$ is a quasi- $\beta$-Banach space with norm $\|\cdot\|$. Suppose $K$ is the modulus of concavity of $\|\cdot\|$. For a mapping $f: X \rightarrow Y$, we define

$$
D f(x, y)=f(x+2 y)+f(x-2 y)-f(x+y)-f(x-y)-3 f(2 y)+6 f(y)
$$

for all $x, y \in X$.

Theorem 3.1 Suppose $f: X \rightarrow Y$ is an odd mapping and $\varphi: X^{2} \rightarrow[0, \infty)$ is a mapping. If there exist a constant $L(0<L<1)$ satisfying

$$
\begin{aligned}
& \|D f(x, y)\| \leq \varphi(x, y), \\
& \varphi(2 x, 2 y) \leq 2^{\beta} L \varphi(x, y)
\end{aligned}
$$

for all $x, y \in X$, then there is a unique additive mapping $A: X \rightarrow Y$ such that

$$
\|f(x)-A(x)\| \leq \frac{1}{6^{\beta}(1-L)} \varphi(0, x)
$$

for all $x \in X$. The mapping $A: X \rightarrow Y$ is defined by

$$
A(x)=\lim _{n \rightarrow \infty} \frac{f\left(2^{n} x\right)}{2^{n}}
$$

Proof Consider the set $\Omega=\{g: X \rightarrow Y\}$ and define a generalized metric on $\Omega$ by

$$
d(g, h)=\inf \{C: C \in \mathbb{R}, C \geq 0,\|g(x)-h(x)\| \leq C \varphi(0, x), x \in X\} .
$$

Then it is not difficult to check that $(\Omega, d)$ is complete. Consider the mapping $\Lambda: \Omega \rightarrow \Omega$ defined by

$$
\Lambda g(x)=\frac{1}{2} g(2 x) .
$$


For any $C$ such that $\|g(x)-h(x)\| \leq C \varphi(0, x)$ for all $x \in X$, we have

$$
\|\Lambda g(x)-\Lambda h(x)\|=\frac{1}{2^{\beta}}\|g(2 x)-h(2 x)\| \leq \frac{1}{2^{\beta}} C \varphi(0,2 x) \leq L C \varphi(0, x) .
$$

Hence $d(\Lambda g, \Lambda h) \leq L C$ and therefore $d(\Lambda g, \Lambda h) \leq L d(g, h)$. Let $x=0$ in (3.1), we have

$$
\|3 f(2 y)-6 f(y)\| \leq \varphi(0, y)
$$

for all $y \in X$. Let $y=x$ in (3.6), we have

$$
\left\|\frac{1}{2} f(2 x)-f(x)\right\| \leq \frac{1}{6^{\beta}} \varphi(0, x)
$$

for $x \in X$. Thus $d(f, \Lambda f) \leq \frac{1}{6^{\beta}}$. According to Theorem 1.1, the sequence $\Lambda^{n} f$ converges to a unique fixed point $A$ of $\Lambda$ on the set $Y=\{g \in X: d(f, g)<\infty\}$, i.e.,

$$
A(x)=\lim _{n \rightarrow \infty} \Lambda^{n} f(x)=\lim _{n \rightarrow \infty} \frac{f\left(2^{n} x\right)}{2^{n}} .
$$

Also we have

$$
A(2 x)=2 A(x)
$$

for all $x \in X$. Since $d(f, A) \leq \frac{1}{6^{\beta}(1-L)}$, we have

$$
\|f(x)-A(x)\| \leq \frac{1}{6^{\beta}(1-L)} \varphi(0, x)
$$

for all $x \in X$ and (3.3) holds true. For all $x, y \in X$, we have

$$
\begin{aligned}
\|D A(x, y)\| & =\lim _{n \rightarrow \infty}\left\|\frac{1}{2^{n}} D f\left(2^{n} x, 2^{n} y\right)\right\|=\lim _{n \rightarrow \infty} \frac{1}{2^{n \beta}}\left\|D f\left(2^{n} x, 2^{n} y\right)\right\| \\
& \leq \lim _{n \rightarrow \infty} \frac{1}{2^{n \beta}} \varphi\left(2^{n} x, 2^{n} y\right) \leq \lim _{n \rightarrow \infty} \frac{\left(2^{\beta} L\right)^{n}}{2^{n \beta}} \varphi(x, y) \\
& =\lim _{n \rightarrow \infty} L^{n} \varphi(x, y)=0 .
\end{aligned}
$$

Hence, $D A(x, y)=0$ for all $x, y \in X$. It follows from Lemma 2.2 that $A: X \rightarrow Y$ is an additive mapping. This completes the proof.

Corollary 3.1 Suppose $X$ is a normed linear space, $\beta=1, \theta$ and $r$ are nonnegative numbers with $r<1, f: X \rightarrow Y$ is an odd mapping and

$$
\|D f(x, y)\| \leq \theta\left(\|x\|^{r}+\|y\|^{r}+\|x\|^{\frac{r}{2}}\|y\|^{\frac{r}{2}}\right)
$$

for all $x, y \in X$. Then there is a unique additive mapping $A: X \rightarrow Y$ such that

$$
\|f(x)-A(x)\| \leq \frac{\theta\|x\|^{r}}{6\left(1-2^{r-1}\right)}
$$

for all $x \in X$. 
Proof For all $x, y \in X$, let $\varphi(x, y)=\theta\left(\|x\|^{r}+\|y\|^{r}+\|x\|^{\frac{r}{2}}\|y\|^{\frac{r}{2}}\right)$. Then the results follows from Theorem 3.1.

Similar to the proof of Theorem 3.1 and Corollary 3.2, we have Theorem 3.2 and Corollary 3.2 whose proofs are omitted.

Theorem 3.2 Suppose $f: X \rightarrow Y$ is an odd mapping, $\varphi: X^{2} \rightarrow[0, \infty)$ is a mapping and there exist a constant $L(0<L<1)$ such that

$$
\begin{aligned}
\|D f(x, y)\| & \leq \varphi(x, y), \\
\varphi\left(\frac{x}{2}, \frac{y}{2}\right) & \leq \frac{L}{2^{\beta}} \varphi(x, y)
\end{aligned}
$$

for all $x, y \in X$. Then there exists a unique additive mapping $A: X \rightarrow Y$ such that

$$
\|f(x)-A(x)\| \leq \frac{1}{6^{\beta}(1-L)} \varphi(0, x)
$$

for all $x \in X$. The mapping $A: X \rightarrow Y$ is defined by

$$
A(x)=\lim _{n \rightarrow \infty} 2^{n} f\left(\frac{x}{2^{n}}\right)
$$

Corollary 3.2 Suppose $X$ is a normed linear space, $\beta=1, \theta$ and $r$ are nonnegative numbers with $r>1, f: X \rightarrow Y$ is an odd mapping and

$$
\|D f(x, y)\| \leq \theta\left(\|x\|^{r}+\|y\|^{r}+\|x\|^{\frac{r}{2}}\|y\|^{\frac{r}{2}}\right)
$$

for all $x, y \in X$. Then there is a unique additive mapping $A: X \rightarrow Y$ such that

$$
\|f(x)-A(x)\| \leq \frac{\theta\|x\|^{r}}{6\left(1-2^{1-r}\right)}
$$

for all $x \in X$.

Theorem 3.3 Suppose $f: X \rightarrow Y$ is an even mapping with $f(0)=0$ and $\varphi: X^{2} \rightarrow[0, \infty)$ is a mapping. If there exists a constant $L(0<L<1)$ such that

$$
\begin{aligned}
& \|D f(x, y)\| \leq \varphi(x, y), \\
& \varphi(2 x, 2 y) \leq 2^{2 \beta} L \varphi(x, y)
\end{aligned}
$$

for all $x, y \in X$, then there is a unique quadratic mapping $Q: X \rightarrow Y$ such that

$$
\|f(x)-Q(x)\| \leq \frac{1}{2^{2 \beta}(1-L)} \varphi(0, x)
$$

for all $x \in X$. The mapping $Q: X \rightarrow Y$ is defined by

$$
Q(x)=\lim _{n \rightarrow \infty} \frac{f\left(2^{n} x\right)}{2^{2 n}}
$$

for all $x \in X$. 
Proof Consider the $\Omega=\{g: X \rightarrow Y: g(0)=0\}$ and define a generalized metric on $\Omega$ by

$$
d(g, h)=\inf \{C: C \in \mathbb{R}, C \geq 0,\|g(x)-h(x)\| \leq C \varphi(0, x), \forall x \in X\} .
$$

Then it is easy to check that $(\Omega, d)$ is complete. Define $\Lambda: \Omega \rightarrow \Omega$ by $\Lambda g(x)=\frac{g(2 x)}{2^{2}}$. If $C$ is a constant such that $\|g(x)-h(x)\| \leq C \varphi(0, x)$ for all $x \in X$, then we have

$$
\|\Lambda g(x)-\Lambda h(x)\|=\frac{1}{2^{2 \beta}}\|g(2 x)-h(2 x)\| \leq \frac{1}{2^{2 \beta}} C \varphi(0,2 x) \leq L C \varphi(0, x),
$$

i.e., $d(\Lambda g, \Lambda h) \leq L C$, hence we have $d(\Lambda g, \Lambda h) \leq L d(g, h)$. Let $x=0$ in (3.9), we have

$$
\|f(2 y)-4 f(y)\| \leq \varphi(0, y)
$$

for all $y \in X$. Thus

$$
\left\|\frac{1}{4} f(2 x)-f(x)\right\| \leq \frac{1}{4^{\beta}} \varphi(0, x)
$$

for all $x \in X$. Hence $d(f, \Lambda f) \leq \frac{1}{4^{\beta}}$. By Theorem 1.1 there is a mapping $Q: X \rightarrow Y$ which is the fixed point of $\Lambda$ and satisfies

$$
d(f, Q) \leq \frac{1}{1-L} d(\Lambda f, f) \leq \frac{1}{4^{\beta}(1-L)} .
$$

Note $Q$ is defined by

$$
Q(x)=\lim _{n \rightarrow \infty} \frac{f\left(2^{n} x\right)}{2^{2 n}}
$$

for all $x \in X$. Similar to the proof of Theorem 3.1, we have $D Q(x, y)=0$ for all $x, y \in X$. Since $Q$ is also even, $Q$ is a quadratic mapping. This completes the proof.

Corollary 3.3 Suppose $X$ is a normed linear space, $\beta=1, \theta$ and $r$ are nonnegative numbers with $r<2, f: X \rightarrow Y$ is an even mapping, $f(0)=0$ and

$$
\|D f(x, y)\| \leq \theta\left(\|x\|^{r}+\|y\|^{r}+\|x\|^{\frac{r}{2}}\|y\|^{\frac{r}{2}}\right)
$$

for all $x, y \in X$. Then there is a unique quadratic mapping $Q: X \rightarrow Y$ such that

$$
\|f(x)-Q(x)\| \leq \frac{\theta\|x\|^{r}}{4\left(1-2^{r-2}\right)}
$$

for all $x \in X$.

Proof The proof is similar to that of Corollary 3.1 and we omit it.

Similar to the proof of Theorem 3.3 and Corollary 3.3, we have the following Theorem 3.4 and Corollary 3.4. 
Theorem 3.4 Suppose $f: X \rightarrow Y$ is an even mapping with $f(0)=0$ and $\varphi: X^{2} \rightarrow[0, \infty)$ is a mapping. If there exists a constant $L(0<L<1)$ such that

$$
\begin{aligned}
\|D f(x, y)\| & \leq \varphi(x, y), \\
\varphi\left(\frac{x}{2}, \frac{y}{2}\right) & \leq \frac{L}{2^{2 \beta}} \varphi(x, y)
\end{aligned}
$$

for all $x, y \in X$, then there is a unique quadratic mapping $Q: X \rightarrow Y$ such that

$$
\|f(x)-Q(x)\| \leq \frac{1}{2^{2 \beta}(1-L)} \varphi(0, x)
$$

for all $x \in X$. The mapping $Q: X \rightarrow Y$ is defined by

$$
Q(x)=\lim _{n \rightarrow \infty} 2^{2 n} f\left(\frac{x}{2^{n}}\right)
$$

for all $x \in X$.

Corollary 3.4 Suppose $X$ is a normed linear space, $\beta=1, \theta$ and $r$ are nonnegative numbers with $r>2, f: X \rightarrow Y$ is an even mapping, $f(0)=0$ and

$$
\|D f(x, y)\| \leq \theta\left(\|x\|^{r}+\|y\|^{r}+\|x\|^{\frac{r}{2}}\|y\|^{\frac{r}{2}}\right)
$$

for all $x, y \in X$. Then there is a unique quadratic mapping $Q: X \rightarrow Y$ such that

$$
\|f(x)-Q(x)\| \leq \frac{\theta\|x\|^{r}}{4\left(1-2^{2-r}\right)}
$$

for all $x \in X$.

Theorem 3.5 Suppose $f: X \rightarrow Y$ is a mapping satisfying $f(0)=0$ and $\varphi: X^{2} \rightarrow[0, \infty)$ is a mapping. If there exists a constant $L(0<L<1)$ such that

$$
\begin{aligned}
& \|D f(x, y)\| \leq \varphi(x, y), \\
& \varphi(2 x, 2 y) \leq 2^{\beta} L \varphi(x, y)
\end{aligned}
$$

for all $x, y \in X$, then there exist a unique additive mapping $A: X \rightarrow Y$ and a unique quadratic mapping $Q: X \rightarrow Y$ such that

$$
\|f(x)-A(x)-Q(x)\| \leq \frac{K^{2}}{2^{\beta}}\left(\frac{1}{2^{2 \beta}(1-L)}+\frac{1}{6^{\beta}(1-L)}\right)[\varphi(0, x)+\varphi(0,-x)]
$$

for all $x \in X$.

Proof If we decompose $f$ into the even and the odd parts by putting $f_{e}(x)=\frac{f(x)+f(-x)}{2}$ and $f_{o}(x)=\frac{f(x)-f(-x)}{2}$ for all $x \in X$, then

$$
f(x)=f_{e}(x)+f_{o}(x)
$$


Then if it is not difficult to check that

$$
\left\|D f_{e}(x, y)\right\| \leq \frac{K}{2^{\beta}}[\varphi(x, y)+\varphi(-x,-y)]
$$

for all $x, y \in X$. Let

$$
\psi(x, y)=\frac{K}{2^{\beta}}[\varphi(x, y)+\varphi(-x,-y)]
$$

for all $x, y \in X$. Then $\psi(2 x, 2 y) \leq 2^{2 \beta} L \psi(x, y)$ for all $x, y \in X$. It follows from Theorem 3.3 that there is a unique quadratic mapping $Q: X \rightarrow Y$ such that

$$
\left\|f_{e}(x)-Q(x)\right\| \leq \frac{1}{2^{2 \beta}(1-L)} \psi(0, x)
$$

for all $x \in X$. Similarly it follows from Theorem 3.1 that there is a unique additive mapping $A: X \rightarrow Y$ such that

$$
\left\|f_{0}(x)-A(x)\right\| \leq \frac{1}{6^{\beta}(1-L)} \psi(0, x)
$$

Hence

$$
\begin{aligned}
\|f(x)-Q(x)-A(x)\| & \leq K\left(\frac{1}{2^{2 \beta}(1-L)}+\frac{1}{6^{\beta}(1-L)}\right) \psi(0, x) \\
& =\frac{K^{2}}{2^{\beta}}\left(\frac{1}{2^{2 \beta}(1-L)}+\frac{1}{6^{\beta}(1-L)}\right)[\varphi(0, x)+\varphi(0,-x)]
\end{aligned}
$$

for all $x \in X$. This completes the proof.

Corollary 3.5 Suppose $X$ is a normed linear space, $\beta=1, \theta$, and $r$ are nonnegative numbers with $r<1, f: X \rightarrow Y$ is a mapping and

$$
\|D f(x, y)\| \leq \theta\left(\|x\|^{r}+\|y\|^{r}+\|x\|^{\frac{r}{2}}\|y\|^{\frac{r}{2}}\right)
$$

for all $x, y \in X$. Then there exist a unique additive mapping $A: X \rightarrow Y$ and a unique quadratic mapping $Q: X \rightarrow Y$ such that

$$
\|f(x)-A(x)-Q(x)\| \leq \frac{5 K^{2}}{12} \frac{\theta\|x\|^{r}}{\left(1-2^{r-1}\right)}
$$

for all $x \in X$.

The proofs of Theorem 3.6 and Corollary 3.6 are similar to that of Theorem 3.5 and Corollary 3.5 and we omit them.

Theorem 3.6 Suppose $f: X \rightarrow Y$ is a mapping satisfying $f(0)=0$ and $\varphi: X^{2} \rightarrow[0, \infty)$ is a mapping. If there exists a constant $L(0<L<1)$ such that

$$
\|D f(x, y)\| \leq \varphi(x, y)
$$




$$
\varphi\left(\frac{x}{2}, \frac{y}{2}\right) \leq \frac{L}{2^{2 \beta}} \varphi(x, y)
$$

for all $x, y \in X$, then there exist a unique additive mapping $A: X \rightarrow Y$ and a unique quadratic mapping $Q: X \rightarrow Y$ such that

$$
\|f(x)-A(x)-Q(x)\|<\frac{K^{2}}{2^{\beta}}\left(\frac{1}{6^{\beta}(1-L)}+\frac{1}{2^{2 \beta}(1-L)}\right)[\varphi(0, x)+\varphi(0,-x)]
$$

for all $x \in X$.

Corollary 3.6 Suppose $X$ is a normed linear space, $\beta=1, \theta$ and $r$ are nonnegative numbers with $r>2, f: X \rightarrow Y$ is a mapping and

$$
\|D f(x, y)\| \leq \theta\left(\|x\|^{r}+\|y\|^{r}+\|x\|^{\frac{r}{2}}\|y\|^{\frac{r}{2}}\right)
$$

for all $x, y \in X$. Then there exist a unique additive mapping $A: X \rightarrow Y$ and a unique quadratic mapping $Q: X \rightarrow Y$ such that

$$
\|f(x)-A(x)-Q(x)\| \leq \frac{5 K^{2}}{12} \frac{\theta\|x\|^{r}}{\left(1-2^{2-r}\right)}
$$

for all $x \in X$.

\section{Competing interests}

The authors declare that they have no competing interests.

\section{Authors' contributions}

All authors carried out the proof. All authors conceived of the study, and participated in its design and coordination. All authors read and approved the final manuscript.

\section{Acknowledgements}

The authors would like to express their sincere thanks to the referees for giving useful suggestions for the improvement of this article. This study was supported in part by the NSF of China (10971117), the NSF of Shandong Province (ZR2012AM024) and the Postdoctoral Science Foundation of Shandong Province of China (201003044).

Received: 9 April 2012 Accepted: 21 June 2012 Published: 2 July 2012

\section{References}

1. Ulam, SM: A Collection of Mathematical Problems. Interscience Publishers, New York (1960)

2. Hyers, DH: On the stability of the linear functional equation. Proc. Natl. Acad. Sci. USA 27, $222-224$ (1941)

3. Aoki, T: On the stability of the linear transformation in Banach spaces. J. Math. Soc. Jpn. 2, 64-66 (1950)

4. Rassias, ThM: On the stability of the linear mapping in Banach spaces. Proc. Am. Math. Soc. 72, 297-300 (1978)

5. Rassias, ThM (ed.): Functional Equations, Inequalities and Applications. Kluwer Academic, Dordrecht (2003)

6. Gajda, Z: On stability of additive mappings. Int. J. Math. Math. Sci. 14, 431-434 (1991)

7. Rassias, ThM, Semrl, P: On the behaviour of mappings which do not satisfy Hyers-Ulam stability. Proc. Am. Math. Soc. 114, 989-993 (1992)

8. Gavruta, P: A generalization of the Hyers-ULam-Rassias stability of approximately additive mappings. J. Math. Anal. Appl. 184, 431-436 (1994)

9. Skof, F: Local properties and approximations of operators. Rend. Semin. Mat. Fis. Milano 53, 113-129 (1983)

10. Kim, H-M, Rassias, JM: Generalization of Ulam stability problem for Euler-Lagrange quadratic mappings. J. Math. Anal. Appl. 336, 277-296 (2007)

11. Park, C: On the Hyers-Ulam-Rassias stability of generalized quadratic mapping in Banach modules. J. Math. Anal. Appl. 291(1), 214-223 (2004)

12. Moslehian, MS, Nikodem, K, Popa, D: Asymptotic aspect of the quadratic functional equation in multi-normed spaces. J. Math. Anal. Appl. 355, 717-724 (2009)

13. Czerwik, S: On the stability of the quadratic mapping in normed spaces. Abh. Math. Semin. Univ. Hamb. 62, 59-64 (1992)

14. Jung, S-M: Hyers-Ulam-Rassias Stability of Functional Equations in Mathematical Analysis. Hadronic Press, Palm Harbor (2001) 
15. Czerwik, S: Functional Equations and Inequalities in Several Variables. World Scientific, Singapore (2002)

16. Park, W-G, Bae, J-H: On a bi-quadratic functional equation and its stability. Nonlinear Anal. 62(4), $643-654$ (2005)

17. Najati, A, Moghimi, MB: Stability of a functional equation deriving from quadratic and additive functions in quasi-Banach spaces. J. Math. Anal. Appl. 337(1), 399-415 (2008)

18. Park, C: The Hyers-Ulam stability of a functional equation deriving from quadratic and cubic functions in quasi- $\beta$-normed spaces. Bull. Sci. Math. 132, 87-96 (2008)

19. Najati, A, Moradlou, F: Stability of quadratic functional equation in quasi-Banach space. Bull. Korean Math. Soc. 45(3), 587-600 (2008)

20. Rassias, JM, Kim, H-M: Generalized Hyers-Ulam stability for additive functional equations in quasi- $\beta$-normed spaces. J. Math. Anal. Appl. 356, 302-309 (2009)

21. Rassias, MJ: Generalised Hyers-Ulam product-sum stability of a Cauchy type additive functional equation. Eur. J. Pure Appl. Math. 4(1), 50-58 (2011)

22. Rassias, MJ: Product-sum stability of an Euler-Lagrange functional equation. J. Nonlinear Sci. Appl. 3(4), 265-271 (2010)

23. Diaz, JB, Margolis, B: A fixed point theorem of the alternative, for contractions on a generalized complete metric space. Bull. Am. Math. Soc. 74, 305-309 (1968)

24. Cadariu, I, Radu, V: Fixed points and the stability of Jensen's functional equation. J. Inequal. Pure Appl. Math. 4(1), 1-7 (2003)

25. Cadariu, I, Radu, V: On the stability of the Cauchy functional equation: a fixed point approach. In: Iteration Theory (ECIT 02). Grazer Mathematische Berichte, vol. 346, pp. 43-52. Karl-Franzens-Universitat Graz, Graz, Austria (2004)

26. Jung, S-M, Rassias, JM: A fixed points approach to the stability of a functional equation of the spiral of Theodorus. Fixed Point Theory Appl. 2008, Article ID 945010 (2008)

27. Park, C, An, JS: Stability of the Cauchy-Jensen functional equation in $C^{*}$-algebras: a fixed point approach. Fixed Point Theory Appl. 2008, Article ID 872190 (2008)

28. Park, C, Rassias, JM: Stability of the Jensen-type functional equation in $C^{*}$-algebras: a fixed point approach. Abstr. Appl. Anal. 2009, Article ID 360432 (2009)

doi:10.1186/1687-1847-2012-98

Cite this article as: Liguang and Jing: On the stability of a functional equation deriving from additive and quadratic functions. Advances in Difference Equations 2012 2012:98.

\section{Submit your manuscript to a SpringerOpen ${ }^{\odot}$ journal and benefit from:}

- Convenient online submission

- Rigorous peer review

- Immediate publication on acceptance

Open access: articles freely available online

- High visibility within the field

- Retaining the copyright to your article 